\title{
Persistent craniopharyngeal canal
}

\section{Canal craniofaríngeo persistente}

\section{Bernardo Carvalho Muniz', Priscilla Haui Fonseca², Bruno Niemeyer de Freitas Ribeiro ${ }^{1,3}$, Emerson Leandro} Gasparetto ${ }^{4}$, Edson Marchiori 5

A two-year-old male, with delayed neuropsychomotor development underwent computed tomography and magnetic resonance imaging (Figure). The diagnosis was persistent craniopharyngeal canal.

Persistent craniopharyngeal canal is a rare congenital anomaly of the skull base and is defined as a well-corticated osseous canal, extending from the roof of the nasopharynx to the base of the sella, over the sphenoid corpus, allowing the pituitary gland to present as a nasopharyngeal mass. Its origin may represent the remnant of the route of Rathke's pouch $^{1}$. Computed tomography and magnetic resonance imaging evaluate the content and limits of the canal well, preventing surgical iatrogenesis, such as hypopituitarism and cerebrospinal fluid leakage ${ }^{2}$.
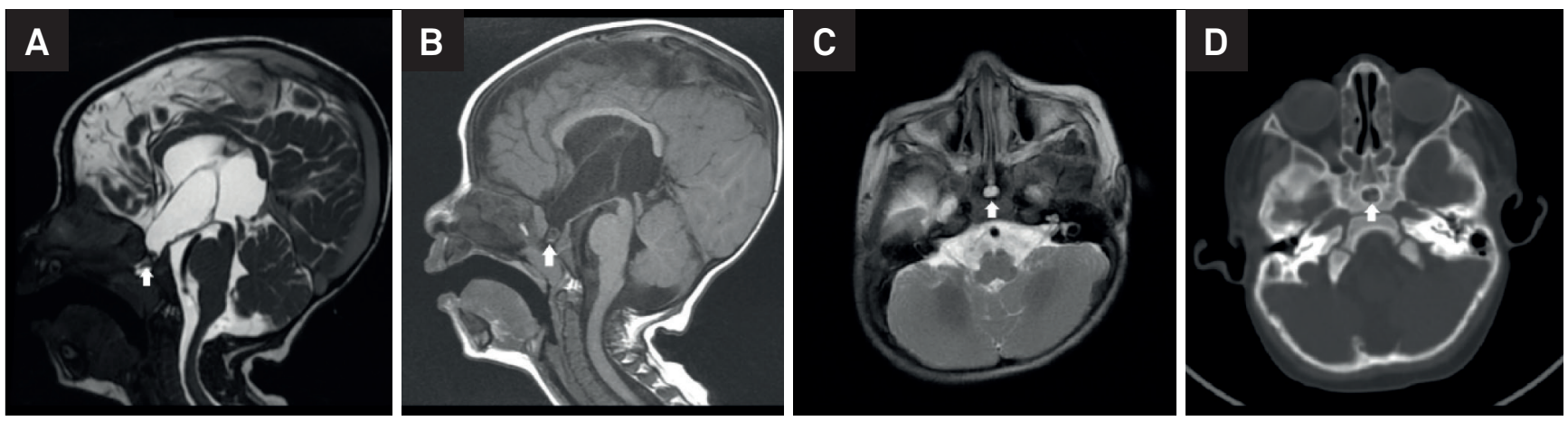

Figure. A: Magnetic resonance, constructive interference in steady state 3D sequence, sagittal plane; B: Magnetic resonance, T1, sagittal plane; C: Magnetic resonance, T2, axial plane; D: Computed tomography, bone window, axial plane. The persistence of the craniopharyngeal canal (arrows in C and D), and the pituitary remnant inside (arrows in A and B). Note that the remnant of the pituitary gland is lower than usual in the nasopharyngeal roof.

\section{References}

1. Akyel NG, Alımlı AG, Demirkan TH, Sivri M. Persistent craniopharyngeal canal, bilateral microphthalmia with colobomatous cysts, ectopic adenohypophysis with Rathke cleft cyst, and ectopic neurohypophysis: case report and review of the literature. Childs Nerv Syst. 2018 Jul;34(7):1407-10. https://doi.org/10.1007/s00381-018-3747-4
2. Mohindra S, Gupta K, Mohindra S. A novel minimally invasive endoscopic repair in a case of spontaneous CSF rhinorrhea with persistent craniopharyngeal canal. Neurollndia. 2015;63(3):434-6. https://doi.org/10.4103/0028-3886.158243

\footnotetext{
IInstituto Estadual do Cérebro Paulo Niemeyer, Departamento de Radiologia e Diagnóstico por Imagem, Rio de Janeiro RJ, Brasil; ${ }^{2}$ Hospital Federal Cardoso Fontes, Departamento de Radiologia, Rio de Janeiro RJ, Brasil; ${ }^{3}$ Clínica 3D Diagnose, Serviço de Radiologia, Rio de Janeiro RJ, Brasil;

«Universidade Federal do Rio de Janeiro, Rio de Janeiro RJ, Brasil;

${ }^{5}$ Universidade Federal do Rio de Janeiro, Departamento de Radiologia, Rio de Janeiro RJ, Brasil.

Correspondence: Bernardo Carvalho Muniz; Instituto Estadual do Cérebro Paulo Niemeyer; Rua do Rezende, 156; 20550-220 Rio de Janeiro RJ, Brasil; E-mail: bernardocmuniz@yahoo.com.br

Conflict of interest: There is no conflict of interest to declare.

Received 16 April 2018; Received in final form 30 July 2018; Accepted 08 August 2018.
} 